\title{
Prenatal Diagnosis of Right Aortic Arch with Mirror Image Branching: Importance of the Three-vessel-trachea View: Editorial Comment
}

\author{
Melih Atahan GÜVENa \\ aClinic of Obstetrics \& Gynecology, \\ Perinatology, \\ Acıbadem Kadıköy Hospital, \\ İstanbul \\ Received: 30.03 .2018 \\ Accepted: 30.03 .2018 \\ Available online: 10.04 .2018 \\ Correspondence: \\ Melih Atahan GÜVEN \\ Acıbadem Kadıköy Hospital, \\ Clinic of Obstetrics \& Gynecology, \\ Perinatology, \\ İstanbul, TURKEY \\ www.melinguven.com
}

An editorial commentary entitled

"Prenatal Diagnosis of Right Aortic Arch with Mirror Image Branching: Importance of the Three-vessel-trachea View" and attributed to the article numbered DOI: 10.5336/gynobstet.2015-48268.

Keywords: Echocardiography; aortic arch syndromes; ductus arteriosus

Copyright $\odot 2018$ by Türkiye Klinikleri he three-vessel (3V) view describes an axial plane that shows the ascending aorta, the main pulmonary artery, and the SVC, at the site where the SVC enters the right atrium. The $3 \mathrm{~V}$ view has an oblique line orientation with the ductal arch, the largest of the three, assuming a more anterior position. The aortic arch is slightly smaller than the ductal arch, in the middle and left to the Trachea, and the SVC, the smallest of the three, is in a more inferior position.

When the course of the aortic arch is toward the right of the trachea, a right-sided aortic arch is present. This anomaly could be distinct or associated with other cardiac malformations. In the right aortic arch, the arch is right sided to the trachea but the abdominal aorta is left sided, unlike cases of isomerism or situs inversus.

A right aortic arch is associated with three main subgroups of arch abnormalities.

1) A right-sided aortic arch can be found in a combination with the left ductus arteriosus, thus forming a U configuration (U-sign) with the trachea in the middle. Such an appearance of a U-sign can also be a sign of double aortic arch, which is a different diagnosis.

2) In the double aortic arch, the aortic arch splits into a right and a left arch to encircle the trachea and esophagus. Both arches merge to form the descending aorta.

3) The pulmonary artery and ductal arch can, on rare occasions, be to the right of the trachea, forming a right-sided V-sign in the 3VT view. This condition can be associated with the right ductus arteriosus and no ring around the trachea and cardiac anomalies are commonly seen in more than $90 \%$ of cases.

In this case, a right-sided Aortic arch with a right Ductus arteriosus and with minor image branching of the Brachiocephalic arteries was seen. This is a mirror image version of the normal left-sided aortic arch. In this anomaly, the left aortic arch distal to the origin of the left subclavian artery 
regresses, resulting in a right aortic arch. The left subclavian and the common carotid arteries merge to form the left brachiocephalic artery, which arises as the first branch of the right aortic arch followed by the right common carotid artery and the right subclavian artery. In almost all cases, the right duc- tus arteriosus persists, whereas the left ductus arteriosus regresses.

In all conditions involving a right-sided aortic arch, the color Doppler is of paramount importance in outlining the course and various ramifications of the vessels. 